\title{
RÉSISTANCE DES CORPUS LONGS ET DÉPLACEMENTS DE LA RECHERCHE: L'EXEMPLE DE LA RÉDACTION CONVERSATIONNELLE
}

\begin{abstract}
A bst ract . De Gaulmyn Marie-Madeleine, Résistance des corpus longs et déplacements de la recherche: l'exemple de la rédaction conversationnelle [Difficulties with a long corpus and the change of the point of gravity in studies: an example of editing a text in the conversational interaction]. Studia Romanica Posnaniensia, Adam Mickiewicz University Press, Poznań, vol. XXV/XXVI: 2000, pp. 109-124. ISBN 83-232-0965-0, ISSN 0137-2475.

In this article the author presents an analysis of empirical material which consists of a recording of interaction between two female students as they edit together a written text on the subject given them. Various characteristics of these students (nationality, social status, upbringing, their past school experiences) cause conflicts which have not been predicted by the researcher and which influence the final form of the text being edited.
\end{abstract}

\section{INTRODUCTION}

Il est devenu impossible de donner une définition unique des recherches comprises dans le vaste champ de l'analyse des discours. Chacun des deux termes analyse et discours posent problème et l'expression entière aussi (Maingueneau 1991, p. 15, recense sept acceptions différentes de discours). L'évolution en 40 ans rend impossible de se référer à Harns, à Althusser, à Foucault, et d'eviter la question de la place de la pragmatique dans la linguistique, voire celle de la linguistique dans la pragmatique (Sperber \& Wilson 1989, Moeschler \& Reboul 1994, Gaulmyn 1997). La diversité des théories de référence, des pratiques de recueil des textes et des méthodologies inclinerait plutôt à penser qu'il existe autant d'analyses de discours et autant de définitions du discours que de chercheurs.

Je préciserai au début de cet article la forme d'analyse de discours que je pratique, l'origine et l'originalité des données étudiées et la nature des objectifs de notre programme de recherche. Mais je me limiterai à l'examen d'un problème particulier né 
de l'analyse elle-même que j'illustrerai sur l'étude d'un corpus. Il arrive en effet que le corpus lui-même opère un déplacement de l'objet de recherche et impose au chercheur une interprétation étrangère au cadre des hypothèses formulées au départ de son étude. L'exposé de ce problème peut conduire à une réflexion plus large, de nature éthique et épistémologique, sur le parcours de chercheur, sur la validité de ses choix et la valeur des résultats, et finalement sur la signification et la légitimité de la recherche elle-même.

\section{L'ÉTUDE DES CONVERSATIONS RÉDACTIONNELLES}

L'analyse des discours, au sens où je l'entends, a subi les effets de l'évolution de la linguistique, qui a intégré dans la langue les règles de l'activité langagière, dites règles de l'actualisation du discours, et ne considère plus seulement les discours produits, mais les processus de production et d'interprétation qui génèrent les discours.

Nous étudions actuellement des corpus longs, authentiques et exhaustifs, enregistrés dans des situations naturelles d'interactions complexes. La recherche entreprise à Lyon par l'équipe du GRIC 2 «Interaction, acquisition et apprentissage des langues» sous la responsabilité de R. Bouchard et M.-M. de Gaulmyn, porte sur une classe particulière d'interactions, dites conversations rédactionnelles où les co-locuteurs sont aussi les co-rédacteurs d'un texte commun (Bouchard 1993, 1996, Bouchard \& Gaulmyn 1997, Bouchard, Gaulmyn \& Sadni-Jallab à paraître 1999). Elles se caractérisent à la fois comme des situations de travail et comme des situations oralo-graphiques. Finalisées par une tâche définie et accomplie pendant l'interaction, comme le sont les réunions de travail de caractère professionnel, elles combinent activités sur de l'écrit et échanges oraux. Les propos échangés accompagnent, guident, contrôlent l'exécution de la tâche qui est la production coopérative d'un texte écrit.

La conversation qui accompagne l'élaboration du texte écrit, des premiers essais à la version finale, est enregistrée au magnétophone. Parallèlement, sont conservés les états successifs du texte écrit, les brouillons avec leurs ratures. Le corpus qui illustre cet article est la rédaction d'un texte argumentatif par deux étudiantes de Français Langue Étrangère, Edita qui est polonaise et Maria brésilienne.

Cette recherche est née de constats liés à l'évolution des recherches linguistiques. La priorité accordée à l'oral pour la description du système phonologique s'est longtemps accompagnée de la mise à l'écart de l'écrit. D'autre part la différence des usages parlé et écrit a été majorée par une double réduction, celle de l'oral aux conversations spontanées et familières, celle de l'écrit à des produits achevés, normés, édités. En réalité il existe une gamme très large d'usages, tant parlés qu'écrits, des aires de recouvrements, et quantité de situations mixtes oralo-graphiques doublement déterminées par les usages parlé et écrit.

Ce projet est né aussi d'un intérêt pour la production de textes écrits. Cette forme d'étude du texte écrit inverse les perspectives habituelles puisqu'elle aborde l'écrit à 
partir du processus oralisé de sa fabrication. Mieux que le texte achevé et définitif, les brouillons, les ratures, les repentirs, les corrections sont les traces de la gestation du texte et des étapes de sa fabrication. En ce sens, notre méthode se rapproche de la critique génétique (Grésillon 1994).

Nouvelle approche du texte écrit, ces situations se veulent aussi une nouvelle approche de l'apprentissage. Si le but des partenaires pendant l'interaction est de produire un texte satisfaisant, le but de la recherche, lui, est de mettre en évidence les processus cognitifs d'apprentissage d'une tâche, exposés et activés par l'interaction qui oblige à verbaliser les contenus cognitifs.

De ce fait, les objectifs linguistiques de ce programme de recherche se doublent d'objectifs didactiques. L'étude linguistique que je poursuis conjoint sémantique de l'énonciation et pragmatique des actes de discours sur un double objet de recherche: d'un côté, le sujet de discours, sujet singulier et multiple, divisé et rassemblé, éclaté et unifié, traversé et modelé par les autres; de l'autre côté, le discours et la (re)formulation, ou la gestation du texte dans le discours, le traitement de la variation dans la stratification du discours lui-même (Gaulmyn 1997).

Il s'agit d'appliquer une linguistique des marques verbales de l'énonciation à l'étude de l'interaction, voir ce que l'observation de ces marques ajoute à une description ethnométhodologique des échanges et à une analyse des actes illocutoires, et voir encore ce que les interactions, la pluralité des locuteurs (à la fois polyphonie et plurivocité), ajoutent à une sémantique de l'énonciation (Vion 1992, 1995, Apotheloz \& Grossen 1995). Il s'agit d'autre part, dans une perspective didactique, d'étudier le processus et les techniques de rédaction, c'est-à-dire les compétences pratiques mobilisées par une tâche d'écriture, et l'impact (positif ou négatif) de la collaboration.

J'ai personnellement souvent fait le constat de la difficulté d'exposer les résultats d'une recherche sur de longs corpus dans le temps d'une communication ou l'espace d'un article. Je dois réduire la masse des données à quelques menus exemples et à la définition d'un seul comportement verbal. L'exemple a son intérêt pour illustrer une règle de l'usage langagier, mais non les régularités propres qui caractérisent le corpus. Pour être valide, la description d'un fait doit être étendue à tout le corpus et vérifiée sur toutes les occurrences. Or ce qui a un sens dans une séquence locale en prend un autre dans un contexte plus étendu et un autre encore dans le contexte de toute la conversation; la signification manifestée par l'un des partenaires pendant l'interaction n'épuise pas les significations que l'analyse peut reconstituer à partir du savoir des partenaires et de son savoir propre. La réflexion sur ces problèmes porte finalement sur la validité d'une analyse des traces linguistiques pour étudier des processus cognitifs et pour décrire la construction interactive d'un événement social dans une situation donnée. Apotheloz et Grossen $(1995,188)$ craignent de courir «le risque d'être en mesure de décrire localement les processus de co-construction du sens, tout en passant à côté de ce qui globalement, c'est-à-dire dans le contexte interactionnel et institutionnel, permettrait de rendre compte de ces activités». 


\section{LE CORPUS EDITA \& MARIA}

Le corpus présenté a été recueilli et transcrit en vue d'un travail de DEA (Omar 1997). L'enregistrement d'l h. 15 de conversation rédactionnelle fournit au chercheur 25 pages de transcription. Le texte écrit est sous deux formes, un brouillon et un texte recopié. Les partenaires sont deux adultes, d'un bon niveau de français, Edita, étudiante polonaise, réservée, pour ne pas dire timide, réputée sérieuse; Maria da Graça, étudiante brésilienne, mère de famille de deux enfants, expansive, chaleureuse. Elles rédigent en collaboration un texte argumentatif qui doit présenter et défendre la position commune qu'elles peuvent choisir entre deux positions contraires. La consigne qui leur a été donnée, commune à plusieurs de nos corpus, est empruntée à A. Giroud (1992): «Vous allez écrire à deux un texte sur les devoirs scolaires à la maison, ce problème des devoirs est largement discuté au niveau de l'école primaire. (...) ceux qui sont contre les devoirs écriront un texte qui a pour titre: halte aux devoirs à la maison, ceux qui sont pour les devoirs écriront un texte qui a pour titre: les devoirs sont nécessaires à l'école primaire».

Le corpus oral comprend 851 «tours de parole» (terme qu'il convient ici de préciser: il ne s'agit pas des interventions constitutives des échanges, mais des changements de locutrice, même s'il y a superposition, chevauchement, simple émission de régulateur, achèvement de l'énoncé commencé), soit 1.132 lignes de transcription. Le texte de 7 phrases et 162 mots, écrit au brouillon par Edita avec 27 ratures, ce qui représente 55 mots barrés, est recopié par Maria. J'ai pu comparer un autre texte rédigé par Maria seule plusieurs jours après sans qu'elle ait eu accès au premier texte, de 121 mots et 6 phrases.

Deux sortes d'hypothèses d'interprétation didactique sont possibles:

1. À elles deux composent-elles un texte différent de ceux que chacune aurait fait seule? Différent veut-il dire meilleur? et de quel point de vue? à quel niveau de rédaction? (correction graphique, correction de langue, registre de langue, «élégance» du style, densité, concision, force des arguments...). Différent veut-il dire pire, stéréotypé, conventionnel, médiocre, proche d'un oral transcrit? Différent veut-il dire composite, disparate?

2. L'explicitation de leurs pratiques rédactionnelles - qui renseigne le linguiste sur ces pratiques intériorisées et disponibles et sur leur métalangage - contribue-t-elle à une prise de conscience de la part des co-rédactrices? Cette distance critique aurait ultérieurement des effets et modifierait leurs pratiques. La situation aurait pour fonction de déstabiliser et de relativiser des pratiques routinières semi-automatiques en modifiant les conditions d'exercice de ces pratiques. C'est la position que je défendrais actuellement.

Deux sortes d'hypothèses proprement linguistiques ont été envisagées:

1. Comment sont négociées par les partenaires les reformulations successives qui donnent naissance aux phrases du texte écrit? Quelles techniques argumentatives, quelles stratégies discursives mettent-elles en oeuvre pour justifier, appuyer ou défendre leurs propositions respectives? Comment se font entre elles les choix et comment se prennent les décisions? 
2. La question centrale à laquelle voudrait répondre l'étude d'un corpus de rédaction d'un texte argumentatif, comme celui d'Edita-Maria, concerne la nature et la forme des productions argumentatives. Nous voudrions savoir si des activités telles que négocier oralement spontanément en face à face, improviser une transaction, s'accorder sur une décision à prendre, relèvent de la même compétence argumentative que la confection d'un texte écrit pour soutenir ou contredire une thèse. Dans ce cas, l'entraînement oral à la discussion argumentée favoriserait la rédaction de textes argumentatifs.

\section{DES TECHNIQUES ARGUMENTATIVES AUX THÈSES EN PRÉSENCE}

Les premiers échanges sont révélateurs de la mise en place des interactants et de la construction mutuelle des rôles. Dès le début de la conversation, avant de se mettre à rédiger à écrire, les deux locutrices donnent leur avis sur la consigne qui vient de leur être communiquée et qui leur propose deux sujets au choix. Maria énonce sa position: elle adopte le second sujet proposé, les devoirs sont nécessaires à l'école primaire, mais soulève une objection justifiée par son expérience familiale de mère d'élève. Ses expressions caractéristiques sont: il y a un problème et par exemple. Sa technique argumentative est donc le recours au cas particulier et la référence à un témoignage vécu.

Exemple 1 (les conventions de transcription sont données à la fin de l'article)

2- M: $\quad h m$ hm 0 il y a un problème hein 0 pour le deux 0 parce que (4) (RIRES) parce que les devoirs à l'école primaire 0 je trouve que c'est nécessaire 0 mais il y a le problème de week-end 0 samedi dimanche 0 parce que les élèves 00 les enfants

3- E: oui

4-M: j'ai des problèmes par exemple avec mon fils 0 parce que: il ne peut pas sortir mercredi 0 après-midi

Edita, qui a laissé Maria s'exprimer avant elle, procède par des formules générales et dogmatiques. L'expression c'est important et les nombreux verbes d'obligation en témoignent.

\section{Exemple 2}

23- E:

euh: ce: qui euh: 00 ce qui est $p=$ plus important je pense euh: c'est que: il faut que les devoirs euh: donnent une certaine liberté aux enfants 0 euh: ça ça doit être 0 déjà le sujet de devoir doit être intéressant 0 pour un enfant 0 pour qu'il fasse euh:

24- M: avec plaisir

25- E: les devoirs avec pl- plaisir 0 devoir euh: doit pas 0 c'est c'est quelque chose d'important 0 mais il doit euh: euh: 0 comment dire euh: enviter $0 \mathrm{en}=$ enviter enviter un élève à la recherche 0 pour qu'il fasse la recherche pour qu'il s'intéresse plus au sujet 0 mais pas euh: devoir ça ne peut pas être quelque chose 0 qui qui euh: je pense des sujets de devoir qui est ennuyeux qui euh: 0 qui est que 0 euh: une sorte de de répétition de ce qu'on a fait pendant le cours 00 /pas/

26- M: /j'ai compris j'ai compris/

27- E: /c'est vrai 0 c'est vrai/ 
Cette comparaison donne une indication sur la différence des deux modes d'expression d'Edita et Maria (dorénavant abrégées en $\mathrm{E}$ et $\mathrm{M}$ ). Encore faut-il montrer que cette différence se retrouve dans la suite de leurs échanges et qu'elles conservent les mêmes structures d'énoncés caractéristiques. Or j'ai constaté que le corpus oral comprend neuf occurrences de l'expression par exemple énoncées par $\mathrm{M}$ et toutes liées à son expérience de mère d'élève, contre deux seulement de $\mathrm{E}$. On peut donc présumer que ce mode d'argumentation habituel chez $M$ est très rare chez $E$. Mais, si l'on s'intéresse aux conditions d'emploi de la même expression par $E$, on constate qu'elle le fait, comme $M$, pour se référer à son expérience personnelle et donner un argument qui a force de témoignage vécu, comme si à un moment décisif elle empruntait à $\mathrm{M}$ une stratégie argumentative. Il s'agit de son expérience de l'enseignement en Pologne sous le régime communiste. Cet emploi de par exemple par $\mathrm{E}$ introduit le tour de parole 115, qui appartient au contexte plus large de l'exemple 11 cité et commenté plus loin. 115-E: par exemple quand c'était en Pologne 0 à cause du communisme 0 on enseignait pas tout... ce qui était important.

Le souci de validation d'une classe de faits très locaux, la reprise de l'expression par exemple, peut mener à dépasser cette classe de faits. La perspective d'analyse est modifiée, puisqu'au lieu d'opposer les formes de langage spécifiques de chacune, on observe comment il y a échange ou contagion entre elles.

D'autre part, si l'on replace les deux extraits comparés plus haut dans leur contexte, ce sont d'autres faits qui s'imposent comme saillants et l'étude s'oriente dans une autre direction.

L'exemple 3 restitue entre les exemples 1 et 2 un passage manquant.

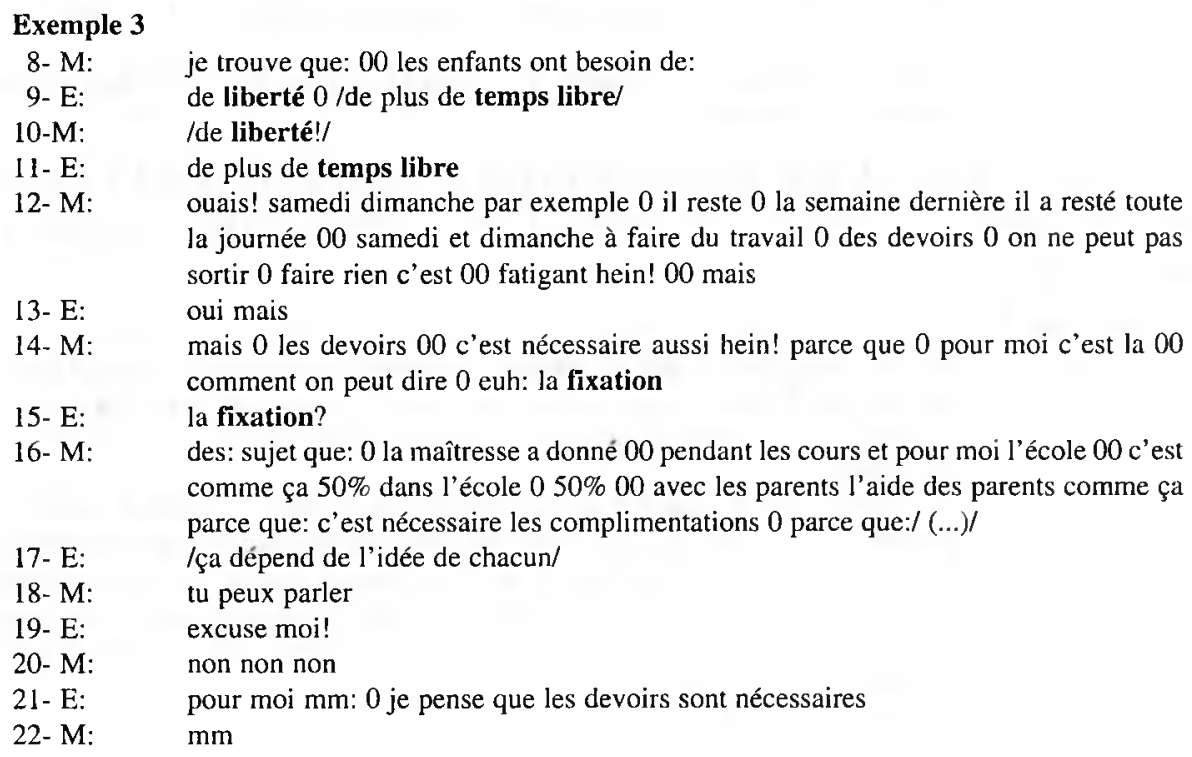


Le mot liberté lancé par $\mathrm{M}$ est reformulé par $\mathrm{E}$ sous la forme temps libre. Puis $\mathrm{E}$ reprend le mot liberté à son compte, et $\mathrm{M}$ en induit celui de plaisir auquel $\mathrm{E}$ substitue alors celui de recherche. $\mathrm{M}$ et $\mathrm{E}$ s'opposent sur la finalité des devoirs à donner aux élèves. C'est la recherche pour $\mathrm{E}$, la fixation pour $\mathrm{M}$, terme qu'elle reformule plus loin par mémorisation et que la phrase 2 du texte écrit reprendra (ll est vrai qu'ils aident à mieux mémoriser tout ce que les élèves ont fait pendant les cours.). Mais cette divergence d'opinion repose sur le sens différent qu'elles donnent au mot liberté. La tension entre elles est sensible aux tours 17 à 19 où $E$ interrompt $M$ pour prendre la parole, et au tour 26 où $\mathrm{M}$ marque une certaine impatience. Cette discussion initiale assez vive semble oubliée dans la suite de leur travail. Pourtant, à la fin de l'enregistrement (tours 715 et sq.), la phrase supplémentaire ajoutée reproduit exactement leur premier échange: Il leur faut une dose de liberté, pour qu'il puisse trouver la joie dans le jeux et dans les recherches personnels.

\section{DES REFORMULATIONS NÉGOCIÉES AU CONFLIT CACHÉ}

Ce corpus devait offrir matière à une étude de l'argumentation orale spontanée. $\mathrm{E}$ et $\mathrm{M}$ devaient négocier les formulations de leur texte commun et parvenir à un accord pour rapprocher leurs positions. Or il a fallu constater que ces négociations étaient le plus souvent implicites ou tronquées. Elles avortent parce que $\mathrm{M}$ s'aligne sur l'avis que E impose avec autorité. À l'exception d'une phrase, les formulations du texte sont toutes proposées par $\mathrm{E}$ et $\mathrm{M}$ se soumet à la compétence rédactionnelle de $E$ qu'elle juge supérieure à la sienne. $M$ suggère sans insister, réagit sans proposer, ou renonce sans insister, tandis que $\mathrm{E}$ formule des assertions, pose et propose les énoncés qu'elle inscrit et relit.

Le corpus offre pourtant deux exemples de négociations longuement développées, mais qui, curieusement, n'ont pas d'effet sur la rédaction du texte. Ces négociations surviennent au moment (toujours crucial dans les situations du même type) où est rédigée la dernière phrase en conclusion du texte: En résumant, le devoir est un outil qui peut servir autant à verifier les connaissances acquises pendant le cours, que pour les amplifier, et parallèlement à répérer des points faibles de chacun des élèves. La première de ces deux négociations naît d'un malentendu d'origine phonétique entre outil et utile. E prononce [util] pour dire outil, avec un emploi métaphorique du substantif, et $\mathrm{M}$ entend utile, adjectif attendu et banal dans le contexte de devoir. C'est une négociation qu'on a envie de dire «inutile», elle piétine indéfiniment alors que le malentendu aurait pu être réglé rapidement, elle tourne presque au sketch comique. Cependant, fait significatif, $M$, dans son texte ultérieur, dès la phrase $n^{\circ} 1$, réemploie outil comme $\mathrm{E}$ l'avait employé (Le devoir est un outil de travail qui approfonde la question traitée dans la salle, parce qu'il fait accomplir un progrès à la réflexion de chaque élève).

L'autre négociation s'engage sur une proposition de $\mathrm{M}$ : 


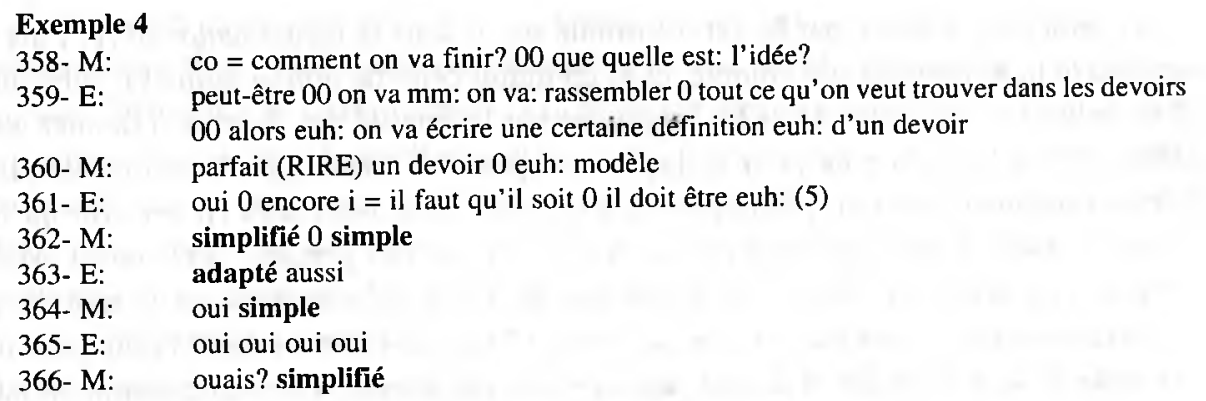

Une vraie négociation (47 tours de parole) s'engage et $\mathrm{M}$, l'auteur de la proposition, s'obstine malgré l'avis contraire de E qui préfère adapté à simple.

\section{Exemple 5}

377- E: c'est 0 mais peut être pas simple mais peut-être adapté 0 à la possibilité aux possibilités des enfants

378- M: donc 0 on peut dire le devoir euh:

379- E: parce que si c'est trop simple $0 \mathrm{j}$ 'ai $\mathrm{j}$ 'ai fait en une minute et ça ça mène à nulle part

Pourtant $\mathrm{E}$ donne un accord verbal à $\mathrm{M}$.

\section{Exemple 6}

391- E:

d'accord 0 alors tu es pour: d'accord 0 on peut dire les devoirs doivent être simplifiés alors? comment tu as mis? simplifiés?

392- M:

oui

393-E:

simplifié euh:

Malgré cet accord E n'inscrit pas le mot dans son brouillon. Il n'en reste dans l'écrit qu'un écho dans un mot phonétiquement associé amplifier, verbe couplé avec vérifier.

Dans son texte ultérieur, $\mathbf{M}$ ne reprend pas non plus ce terme, ni l'idée - qui lui tenait à coeur - de simplifier pour l'élève le travail à faire à la maison. Aucune trace non plus de l'idée - à laquelle elle tenait beaucoup pendant l'interaction - d'éviter la surcharge des élèves. C'est plutôt l'inverse: elle parle dans son nouveau texte de réflexion approfondie, synthèse, contrôle sérieux, termes qui semblent plus proches des thèmes exposés par $\mathrm{E}$ que de ceux défendus par $\mathrm{M}: \mathrm{Si}$, pendant le travail commun, on peut parler de la soumission de $\mathrm{M}$ à l'autorité de $\mathrm{E}$, dans son travail individuel, on peut parler d'une réelle influence exercée par $E$ sur $M$.

L'étude des négociations conduit donc à des questions imprévues au départ sur la forme de l'interaction et ses effets sur l'évolution des opinions. Ce qui est le plus longuement négocié n'importe pas au texte écrit commun, mais cette discussion a des effets ultérieurs dans une écriture individuelle.

Par ailleurs, dans cette négociation sur simple, simplifié, resurgit aussi, de façon inattendue (pour le chercheur qui analyse la transcription), le mot de recherche. Une intervention de $M$ (tour 374 ) pose en contexte un problème de cohérence. 


\section{Exemple 7}

373- E: $\quad \mathbf{~ i ~ m ~} \mathbf{p} \mathbf{l}$ i f i é si le devoir est trop s i m p l e ça dépend du motivation des élèves 0 $\mathrm{s}$ il y a de $=$ des élèves qui reçoivent que des devoirs simples 0 ils euh:

374- M: tu penses euh: tu penses euh: au devoir au plan de de la recherche 0 c'est ça?

$\mathrm{M}$ interprète les réticences de $\mathrm{E}$ à accepter son idée de devoir simplifié par le retour implicite de la préoccupation de $\mathrm{E}$ au sujet de la recherche. Toujours conciliante, $\mathrm{M}$ tente de rapprocher recherche et simplifiée (on retrouve ses expressions familières c' est un problème et par exemple)

\section{Exemple 8}

386- M:

c' est un problème qui 00 difficile pour régler hein! 00 mais $00=$ on peut directionner les devoirs 0

387- E: oui

388- M: euh: au plan de 0 d'une recherche 0 mais d'une recherche 0 euh: simplifiée

389- E: $\quad h m$

390- M: parce que 0 par exemple 0 le professeur donne des recherches mais euh: les élèves besoin: de de lire 0 de 00 de faire des photocopies 0 de organisation 0 une fois deux fois ça va 0 toujours il y a 0 beaucoup de choses à faire.

C'est à cette proposition de $\mathrm{M}$ que $\mathrm{E}$ semble donner son accord (tours 391-393). La suite montre que l'enjeu de leur discussion n'est pas tant la nature trop facile ou trop difficile des devoirs donnés aux élèves que la finalité de ces devoirs: vérification du travail fait en classe pour $M$ ou recherche libre et personnelle de l'élève pour $E$.

\section{Exemple 9}

394- M: $\quad$ simplifié ça veut dire 0 euh: qui donnent les devoirs 0 mềme au plan de la recherche 0 mais 00 objectif 00

395- E: devoirs objectif

396- M: pour pour simplifier 0 simplement pour la mémorisation pour la fixation pour savoir si: si l'élève 0 il a compris 0

397- E: ah oui!

398- M: parce que c'est nécessaire

399- E: oui d'accord 0 parce que:

400- M: parce que par exemple 0 quand mon fils il fait des les devoirs 0 même nous:

401- E: oui

402- M: c'est à cause de devoir que on va savoir si on a compris ou non

(...) 406-M: quand on fait le devoir tout seul 0 c'est a la vérification 00 de chaque problème.

407. E: d'accord

Toutes les fois que M s'enflamme pour une thèse, elle recourt à l'argument de son expérience familiale qui lui donne sur le sujet une compétence supérieure à celle de E. Si E n'inscrit pas le mot simplifié, elle enregistre la notion de vérification dans la phrase.

Le constat de cette attention de $\mathrm{E}$ aux idées de $\mathrm{M}$ rend plus délicate l'interprétation de leur mode de collaboration. $L$ 'absence de reformulations négociées incline à penser que E domine la situation, formule et rédige seule les phrases. Mais elle donne forme écrite à des idées qui viennent de M. D'autre part, comme elle tient à ses propres thèses, elle juxtapose dans le texte d'autres phrases, au prix d'une discontinuité 
et d'un effet de «collage». L'entente entre $\mathrm{E}$ et $\mathrm{M}$ est plus apparente que réelle et leur collaboration n'a pas un effet positif sur la composition et la rédaction du texte. Elles défendent toutes les deux la thèse de la nécessité des devoirs à la maison, mais elles s'opposent au sujet de la fonction et donc de la nature des devoirs.

\section{L'HISTOIRE D'UN CONFLIT NON RÉSOLU}

Le corpus a imposé à la recherche une nouvelle direction, à savoir l'étude du mot recherche lui-même, le retour d'un mot qui témoigne de la permanence d'un conflit latent, non résolu. Ce conflit laisse des traces dans le texte produit et il a des effets sur la qualité de la rédaction.

Le mot recherche(s) lancé par Edita au début de leur conversation (tour 25, exemple 2) est répété 45 fois à l'oral ( 31 par E, 14 par M). Le même mot est inscrit 3 fois sur le brouillon, raturé 2 fois, à cause de la suppression d'une phrase: ils peuvent être une sorte d'introduction à la recherche, et par substitution du mot développement. Il n'est conservé qu'une seule fois dans le texte final, grâce à la phrase supplémentaire insérée au moment de recopier le texte: Il leur faut une dose de liberté, pour qu'il puisse trouver la joie dans le jeux et dans les recherches personnels. Il réapparaît une fois dans le texte que Maria écrit seule quelques jours plus tard: Il peut avoir lieu avec d'autres études explorant d'autres voies de recherche individuelle.

Les mots rencontrés dans le contexte de recherche sont à l'oral: liberté 15 fois, libre 3 fois; restreindre 17 fois; simple 7 fois, simplifié 12 fois; équilibre 23 fois (mais seulement au début de la conversation, avant le tour 293); individuel 11 fois, personnel 6 fois. De ces mots ne sont conservés dans le texte écrit que liberté, restreindre, individuel et personnel (ce dernier 2 fois), grâce à la phrase 3 (Cependant parfois, les devoirs trop dirigés peuvent restreindre la possibilité du developpement plus individuels, alors plus adaptés aux besoins personnels des élèves.) et grâce à la phrase 5 supplémentaire citée plus haut.

À l'occasion de la discussion sur simple, simplifié, la suggestion de $\mathrm{M}$ au tour 374 révèle que cette question de la recherche continue à les opposer. Le conflit non réglé au début - mais gommé par politesse et par l'abandon de $\mathrm{M}$ - resurgit à cette occasion comme à beaucoup d'autres. Le désaccord initial entre $\mathrm{E}$ et $\mathrm{M}$ au sujet de la finalité des devoirs à la maison se manifeste à propos du mot recherche, et ce mot, associé à ceux de libre, liberté, court comme un fil rouge d'un bout à l'autre du corpus.

Toute l'interaction est concernée. Dans l'exemple 2 du début de la conversation, cité plus haut, E réagit contre l'argument de M pour l'utilité des devoirs (tour 14 la fixation), et expose sa position (tours 23-25: un devoir intéressant invite à une recherche, un exercice de pure répétition du cours est ennuyeux), position qu'elle maintient (dans les tours 29,31,37, reviennent liberté, sujet plus libre, recherche personnelle) malgré les réserves de $\mathrm{M}$ (qui parle aux tours 30 et 34 de la mémorisation). 
C'est au moment de rédiger au brouillon la phrase 2 que $\mathrm{M}$ reformule le thème tel que l'avait lancé E en l'attribuant explicitement à E (tour 78).

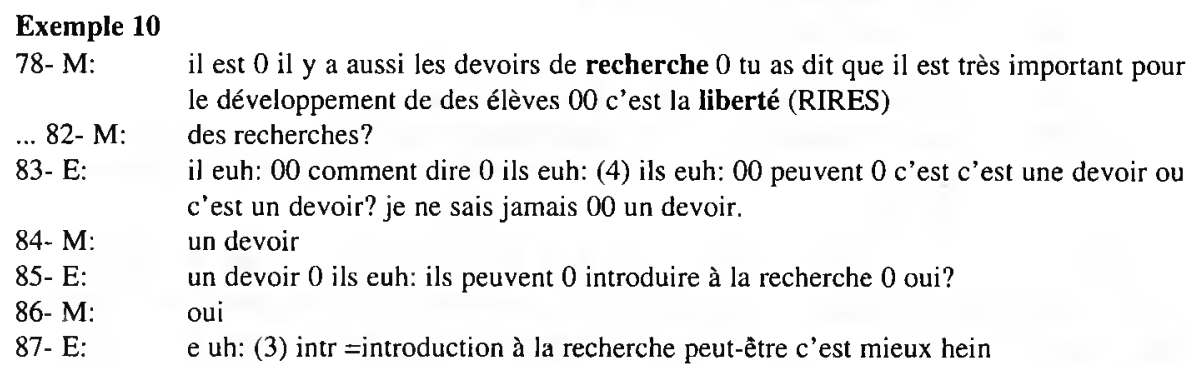

Cette deuxième phrase, ils peuvent être une sorte d'introduction à la recherche, sera supprimée ensuite du texte final. E, qui a proposé une rédaction de la phrase 2 (tour 87), élabore une suite virtuelle qui deviendra la phrase 3. S'ouvre alors une séquence d'explication qu'on pourrait dire latérale, mais qui est effectivement centrale pour comprendre la valeur que $\mathrm{E}$ donne à recherche et à liberté. C'est là que $\mathrm{E}$ reprend l'expression par exemple.

\section{Exemple 11}

109-E: tu sais euh: je j'ai j'ai lu quelque part que en Pologne et euh: euh: les enfants ont beaucoup plus de cours que 0 d'heures de cours que aux États-Unis ou en France

$111-\mathrm{E}$ (...) et ça veut pas dire que que les que les américains sont sont euh: euh: moins éduqués que les polonais

113- E: (...) et en plus 0 si encore l'éducation euh: $m$ : est menée à la manière libérale ça veut dire on enseigne tout tout ce qu'il faut enseigner tout ce qu'il faut savoir de de enseigne de la culture générale

115- E: (...) qui vient du monde entier 0 qu'on est pas crispé euh: quand 0 par exemple quand c'étaiten Pologne 0 à cause du communisme 0 on enseignait pas tout 0 on enseignait ce que ce qui ce qui était euh: euh: ce qui était comment dire euh: euh: ce qui était important euh: pour pour pour élever les enfants dans dans cette dans cette ambiance de communisme 0 alors c'est (...) tu l'as entendu

116- M: ouais ouais

117- E: alors je pense que euh: ici le devoir 0 si le devoir euh: envite à la recherche et et touche les problèmes plus général et et très large 0 ça va 0 mais si les devoirs sont sont euh: sont fixés euh: sur un but trop restreint 0

118- M: oui

119- E: $\quad$ ça ça peut 0 ça peut $=00$ tu tu me comprends?

L'élaboration de la phrase 3 qui traite négativement du thème de la liberté dure très longtemps. Cette phrase a d'abord au brouillon la forme Cependant parfois les devoirs trop dirigés peuvent restreindre la possibilité de recherches plus individuels. E élabore le début de la phrase, se le dicte à voix haute au fur et à mesure qu'elle l'inscrit ou va l'inscrire. (Les énoncés prononcés sur le ton de la dictée sont entre crochets). Elle est en train 'de chercher un complément au verbe restreindre et a essayé plusieurs mots sans tenir compte d'une proposition de M, l'expression liberté de culture, que $\mathrm{M}$ reformule par la liberté de choisir (tour 148). E reprend bien le mot liberté mais l'associe à celui de recherche, ce que M conteste (tour 150). 


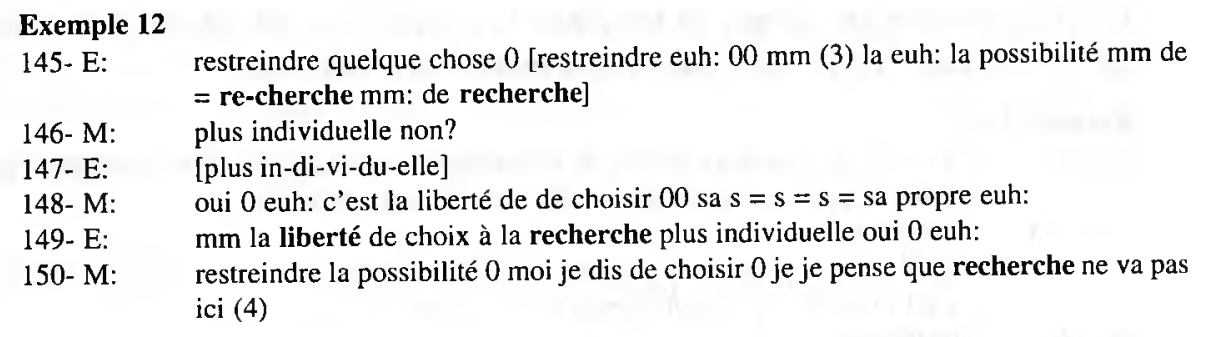

Ce n'est qu'en relisant la phrase pour la recopier que le terme de recherche, qui a été inscrit par $\mathrm{E}$ malgré la réserve de $\mathrm{M}$, est raturé et remplacé par l'expression développement global expression proposée par $\mathrm{E}$, et précisée ensuite par les besoins personnels: l'idée sous-jacente est de favoriser l'épanouissement personnel des élèves, thèse sur laquelle $\mathrm{E}$ et $\mathrm{M}$ peuvent parfaitement s'accorder.

\section{Exemple 13 \\ 617-E:}

mais ici 0 mais je je j'hésite 0 je pense qu'on va mettre euh: LIT cependant parfois 0 euh: les devoirs peuvent restreindre la possibilité 0 les devoirs /trop dirigés peuvent restreindre la possibilité de recherche plus individuelle/ +

618- M: LIT /trop dirigés peuvent restreindre la possibilité de recherche plus individuelle/t

619- E: la possibilité peut être 0 de du développement 00 euh:

620- M: ah oui ouais

621- E: oui

622- M: parce que: (...) je n'ai pas envie de recherche

Avant de relire tout le brouillon pour le recopier, le mot de recherche avait resurgi pendant l'élaboration de la phrase de conclusion. La négociation sur simple, simplifié étudiée précédemment relance la discussion sur recherche. M proposait simplifié, simple (tour 362, exemple 4). E se livre à une série d'objections de plus en plus explicites contre la proposition de $M$ (voir exemples 4 et 7 ) et $M$ comprend très bien où $E$ veut en venir (ou plutôt en revenir) (tour 374 , exemple 7 , tu penses euh: tu penses euh: au devoir au plan de de la recherche 0 c'est ça). L'aboutissement de cette discussion interrompue est l'ajout d'une phrase intercalée entre deux phrases déjà écrites au brouillon que $\mathrm{M}$ est en train de recopier. E a proposé de façon autoritaire d'ajouter une autre phrase et en commence immédiatement la rédaction (tour 706 il euh: il faut leur laisser 0 euh:), initiative que $M$ conteste à cause de la liaison logique des phrases du brouillon (tour 707 il faut (...) parce qu'on a écrit il ne faut pas donc). Effectivement, la structure syntaxique de la phrase précédente (Il est certain que..., mais il ne faut pas que...) appelait naturellement la phrase qui suivait (Donc, il faudrait que...). En intercalant une nouvelle structure positive, avec une autre construction syntaxique du même verbe (Il leur faut une dose...), l'enchaînement est brisé.

\section{Exemple 14}

715- M:

716- E:

qu'est ce que tu veux dire? rapport il faut 0 euh: $0 j=$ je veux dire tout simplement qu'il faut leur laisser 0 un peu de liber de de liberté
(...)722-E: Ion n'a pas parlé de recherche non plus/

je je voulais je voulais dire que il faut pas que les enfants soient trop surchargés par 
723- $\mathrm{M}: \quad$ non non non plus

724- E: $\quad$ (...) oui 0 alors euh: il leur faut un certain euh: euh: liberté attends

725- M: hm

726- E: $\quad \mathrm{j}=\mathrm{je}$ vais écrire peut-être ici 0 il 0 leur 0 euh: il leur ils ont besoin peut-être non (...) [il leur faut une cer-taine euh: li-berté]

727- M: oui 0 ça ne marche pas 0 parce que on a écrit certain 0 certain

728- E: ah oui! d'accord oui oui d'accord il leur faut euh: quel est le synonyme de certain euh.: une alors oui une dose 0 dose 0 dose de liberté 0 euh: pour qu'il euh: $\mathrm{mm}$ : euh: $\mathrm{mm}$ : euh: pour leur donner l'occasion de recherche personnelle non? 0 pour leur donner l'occasion euh: $00 \mathrm{~mm}$ : non 0 parce qu'on n'a pas 0 on a omette cette phrase

729- M: oh oui

730- E: on a omis cette phrase 00 euh:[il leur faut une 00 euh: dose de liberté]

(...)740- E: de recherche on a parlé de recherche personnelle (...) de: euh: mm: de recherche oui?

741-M: $\quad$ euh 00 on peut (...) les recherches 0 pour: pour son $\mathbf{p}=$ plaisir (3)

742- E: ou il faut une dose de liberté pour euh:

743- M: avoir

744- E: pour: euh: qu'ils puissent trouver la j o i e dans: (RIRE)

745- M: ouais pourquoi pas

746- E: oh! je ne sais pas euh: [pour qu'ils puissent trouver 0 la j oi e euh: dans les jeux 0 les

747- M: d'accord

L'élaboration de la phrase par $\mathrm{E}$ se fait rapidement. Un problème de répétition d'un mot déjà employé ailleurs est soulevé par $\mathrm{M}$ (tour 727 sur certaine) et résolu. E rappelle que la phrase 2 initialement écrite dans le brouillon a été omise (tour 728), ce qui justifie l'insertion d'une autre phrase sur la recherche, et elle trouve la formule de compromis (tours 742-746) qui concilie la joie dans les jeux et les recherches personnelles, en intégrant la suggestion de plaisir faite par $\mathrm{M}$.

Par comparaison avec les autres phrases du texte, on peut dire que $\mathrm{E}$ a mené rondement son affaire et qu'elle a réussi in fine à récupérer ce terme de recherche qui lui tenait à coeur.

\section{L'INTERPRÉTATION DU CONFLIT}

Ce conflit latent entre $\mathrm{E}$ et $\mathrm{M}$ permet de comprendre les remords (suppression de le phrase 2), les ratures (phrase 3), les ajouts (phrase 5) et les incohérences du texte écrit, mais il confère aussi une cohérence au corpus oral. Au delà du terme de $r e$ cherche, l'enjeu tient à la différence de sens donnée au terme de liberté par la Polonaise qui a connu l'absence de liberté et par la Brésilienne qui n'en a jamais manqué. E corrige le mot liberté lancé par $\mathrm{M}$ et le reformule par temps libre pour des loisirs en famille (tours 4-12), ce qui est l'acception donnée par M. En parlant de liberté, E veut signifier la liberté d'expression d'une éducation libérale affranchie de la censure politique (tours 109-119, exemple 4).

Ce n'est qu'au début de l'interaction que l'écart des deux positions est clairement expliqué et les reprises du mot recherche sont toujours des rappels de cet échange 
initial sur la fonction que les devoirs doivent remplir. Mais, si l'on élargit le contexte d'interprétation au delà de la transcription des échanges de travail entre $\mathrm{E}$ et $\mathrm{M}$, le thème et le terme de recherche font vraisemblablement écho à la présentation de la situation qui a été faite à $\mathrm{E}$ et $\mathrm{M}$ : le travail demandé était une collaboration à une recherche universitaire et l'enregistrement des données dont une étudiante avait besoin pour son mémoire de «recherches». Un élément de la situation externe se reflète à l'intérieur du texte à produire alors qu'il était censé ne pas intervenir dans le protocole et il témoigne de la réflexivité du cadre externe de l'énonciation sur la tâche d'écriture. À quoi s'ajoute une réflexivité supplémentaire, puisque j'ai choisi d'illustrer par ce corpus de recherche l'exposé d'une «recherche» sur la valeur de mes «recherches».

Mais l'interprétation doit aussi envisager les effets sur $M$ de ce conflit. Dans le texte rédigé plusieurs jours après par $M$ seule sans accès au premier texte, se trouve la phrase: L'exercice bien dosé par les enseignants peut avoir lieu avec d'autres études explorant d'autres voies de recherche individuelle. Elle réemploie d'elle-même l'expression recherche individuelle, alors qu'elle a passé toute l'interaction à la contester et en tous cas à l'attribuer en propre à E. Ce qui était une hétéro-reformulation pendant leur interaction devient une auto-reformulation, ou presque: $M$ adopte le mot, mais elle marque sa distance (d'autres études explorant d'autres voies: l'idée adoptée par elle vient décidément de l'autre).

Le texte de $\mathrm{M}$ reprend les idées défendues par $\mathrm{E}$ et des termes de $\mathrm{E}$ (outil, recherche individuelle). $\mathrm{M}$ a abandonné ses idées personnelles et reformule et transforme les idées empruntées à $E$ avec des termes nouveaux. Pendant l'interaction, M paraissait soumise et en état d'infériorité, mais elle en a probablement tiré profit. Elle a rédigé après avoir discuté avec $E$ un texte personnel sûrement différent et probablement meilleur que celui qu'elle aurait écrit sans l'interaction avec $\mathrm{E}$.

\section{CONCLUSION}

Pour ce corpus, il s'est produit un déplacement vers un objet d'étude imprévisible au départ. Sous le travail régulier et continu de rédaction, s'est révélée l'existence d'un travail secret, parallèle, discontinu, d'un souci qui resurgit avec insistance. Activité secondaire, ou facteur déterminant? C'est le partenaire fort (Edita) qui en a l'initiative, mais elle le fait «comprendre» (mot de Edita à Maria), partager et finalement adopter par l'autre.

Faut-il parler de la résistance des corpus longs à l'étude linguistique ou de l'attirance du chercheur pour ces corpus? Nos corpus sont d'inépuisables réservoirs d'exemples. Ils permettent d'illustrer la grammaire des formes discursives dans la confrontation et le va-et-vient des usages langagiers du parlé spontané et de l'écrit académique qui, dans le cas d'Edita-Maria, relèvent de l'usage d'une langue seconde apprise. Mais chaque corpus pris dans sa situation particulière possède une signification singulière, originale comme l'est chaque fois la texture des voix et le tissage des paroles, chaque corpus possède son unité propre et ses principes d'organisation. 
Aussi les corpus résistent aux généralisations que le chercheur voudrait en tirer par comparaison. D'autre part le chercheur n'est jamais assuré d'avoir choisi la meilleure des interprétations. Le corpus change selon l'angle de vue du chercheur. Malléable, le corpus offre à chacun et chaque fois le dessin d'un nouveau paysage. À quel «grain» de finesse l'analyse doit-elle se situer? Suivant la distance qu'il prend vis-à-vis de son corpus et la taille des objets qu'il observe, le chercheur ne voit pas les mêmes faits. C'est l'intérêt pris à l'étude du corpus qui l'entraîne vers une suite d'interprétations qui dépassent le cadre esquissé au départ et qui excèdent peut-être la compétence du linguiste, mais répondent au désir qu'il a de donner sens à sa recherche.

La question initiale à laquelle voulait répondre l'étude du corpus touchait à l'argumentation dans ses formes écrites rédigées et dans ses techniques orales en face à face. Une autre question qui s'est imposée au cours de l'étude de ce corpus est la mise en relation du processus de travail avec les capacités d'évaluation qualitative du produit par leurs auteurs et leurs capacités d'amélioration de ce texte. Quels modèles textuels guident la fabrication de l'objet-texte? Quelles représentations de la norme d'évaluation qualitative du texte produit ont été intériorisées? Quelle influence a le fait que $E$ et $M$ soient non-natives ? Et finalement quels effets - positifs et négatifs - entraîne la collaboration?

Ces objectifs de recherche sont légitimes. Cependant il se trouve que l'enjeu de l'interaction réside ailleurs, il réside dans le désaccord initial et fondateur qui permet aux partenaires d'exprimer, à propos d'un thème de rédaction imposé de l'extérieur et au cours d'une tâche quasi-scolaire, la vérité et l'irréductibilité de leurs expériences personnelles. L'opposition exprimée par Maria et Edita sur la nature des devoirs à la maison (révision du travail de la classe pour $\mathrm{M}$, invitation à des recherches personnelles pour $\mathrm{E}$ ) manifeste la différence entre la mère de famille d'écoliers et l'étudiante célibataire, mais révèle la signification différente qu'elles attachent à la liberté (temps de loisir pour M, liberté de pensée pour E). Cette opposition n'exclut pas l'influence réciproque qu'elles exercent l'une sur l'autre. E s'applique à mettre en mots et en phrases les idées suggérées par M. Et M, lorsqu'elle écrit seule quelques jours plus tard, montre que les mots et les idées de E sont devenus les siens.

L'analyse sémantique et pragmatique et son orientation didactique débouchent en fin de compte sur une explication psychologique et littéraire, plus thématique que lexicale. D. Maingueneau (1990 et 1991 p. 10), citant A. Culioli et P. Favre, note que l'analyse de discours en France prolonge et se nourrit de la pratique de l'explication des textes littéraires. L'analyse de discours a surtout changé de méthode et d'objet. Cet article montre le glissement d'une analyse des structures morpho-syntaxiques locales à une étude sémantique et pragmatique des formes lexicales qui ont une fréquence significative dans l'ensemble du corpus, et le déplacement d'une description linguistique à une interprétation psychologique dont la validité n'excède pas les limites du corpus. On peut y voir un retour de la linguistique structurale vers l'analyse littéraire, ou l'évolution qui se dessine actuellement des Sciences du Langage aux Sciences de la Cognition. 


\section{CONVENTIONS DE TRANSCRIPTION}

$\begin{array}{ll}= & \text { élision } \\ : & \text { allongement } \\ 0 & \text { pause d'une seconde, } 00 \text { deux secondes, }(\mathrm{x}) \mathrm{x} \text { secondes } \\ / / & \text { chevauchement des tours de parole } \\ \text { l e t t r e s } & \text { e s p a c é e s pour articulation lente } \\ (\ldots) & \text { passage difficilement audible } \\ (\mathrm{xxx}) & \text { interprétation possible d'un passage mal audible } \\ ? & \text { intonation de question } \\ ! & \text { intonation d'exclamation ou exagération }\end{array}$

MAJUSCULE: phénomènes paratextuels dont la fin est indiquée par + (MAJUSCULE) phénomène paratextuel ponctuel

Sont en caractères gras les termes sur lesquels porte l'analyse de l'exemple.

Les phrases du texte écrit sont en italiques. L'orthographe est conservée. Les mots raturés sont en lettres barrées.

\section{BIBLIOGRAPHIE}

A potheloz D., Grossen M. (1995), L'activité de reformulation comme marqueur de construction du sens: réflexions théoriques et méthodologiques, in: L. Mondada éd., Formes linguistiques et dynamiques interactionnelles, Cahiers ILCF, Lausanne, 177-195.

Bouchard R. (1993), Interaction et processus de production écrite: une étude de pragmatique impliquée, Halté J.F. dir. Interactions: l'interaction, actualité de la recherche et enjeux didactiques, Metz, Casum.

B ou chard R. (1996), Compétence argumentative et production écrite en langue étrangère et maternelle, Langue française, 112, 88-104.

B ou chard R., Gaulmyn M.-M. de (1997), Médiation verbale et processus rédactionnel: parler pour écrire ensemble, in: M. Grossen \& B. Py ed. Pratiques sociales et médiations symboliques, P. Lang, 153-173.

Bouchard R., Gaulmyn M.-M. de, Sadni-Jallab R. (1999), à paraître Impeccable...!!!! Le processus d'amélioration du texte en écriture coopérative, Cahiers de Linguistique Française, Genève.

Gau lmy n M.-M. de (1997), Sémantique de l'énonciation et pragmatique des actes de discours: une jonction devenue nécessaire, Revue de Sémantique et Pragmatique, Orléans, $n^{\circ} 2,105-122$.

Girou d A. (1992), Écrire à deux un texte argumentatif en français langue étrangère: processus de production, Mémoire de recherche, Université Genève.

Gresillon A. (1994), Éléments de critique génétique: lire les manuscrits modernes, Paris, Puf.

Maingueneau D. (1990), Pragmatique pour le discours littéraire, Bordas, Paris.

Maingue ne a D. (1991), L'analyse du discours, Hachette, Paris.

Moeschler J. \& Reboul A. (1994), Dictionnaire encyclopédique de pragmatique, Seuil, Paris. Omar H. (1997), Mémoire de DEA de Sciences du Langage, Université Lumière Lyon 2.

Sperber D. \& Wilson D. (1989), La pertinence. Communication et cognition. Minuit, Paris.

Vion R. (1992), La communication verbale, Hachette, Paris.

Vion R. (1995), La gestion pluridimensionnelle du dialogue, Cahiers de Linguistique française, 17 , Genève. 